\title{
DETECCIÓN DE CONDUCTAS AGRESIVAS "BULLYINGS" EN ESCOLARES DE SEXTO A OCTAVO AÑO, EN UNA MUESTRA COSTARRICENSE
}

\author{
Hannia Cabezas Pizarro \\ Profesora de la Escuela de Orientación y Educación Especial, \\ Facultad de Educación, Universidad de Costa Rica, \\ San José, Costa Rica.
}

\begin{abstract}
Resumen: El presente trabajo es un estudio piloto llevado a cabo con una muestra de estudiantes costarricenses, que se encuentran entre sexto y octavo año, que pretende medir las conductas agresivas que se presentan en esa población. Generalmente ubicamos estas conductas fuera del contexto escolar, sin embargo, es un hecho que se vive en nuestras aulas con más frecuencia de la que imaginamos, situación que se está tornando preocupante y a la que debe prestarse atención. Los datos obtenidos en la muestra nos indican porcentajes de conductas agresivas en este grupo comparables con la media europea, situación que no debe escapar a la atención de las personas encargadas de disciplinar y orientar a esa población.
\end{abstract}

Palabras clave: Bullying, victimización, abuso de poder, acoso escolar.

\begin{abstract}
This paper is a pilot study based on a sample of Costa Rican students who are between sixth and eighth grade; this study seeks to measure aggressive behavior in this population. Generally we observe this behavior outside the school context; however, this situation is an increasingly frequent reality in our classrooms, a situation that is becoming a concern which demands our attention. The data in our sample indicates percentages of aggressive behavior in this group that can be compared to the European standard measure, a situation that should be focused by the people in charge of this population's discipline and guidance.
\end{abstract}

Key words: Bullying, victimization, power abuse, school harassment.

\section{Introducción}

Cuando hacemos referencia en Costa Rica, a conductas agresivas o a situaciones violentas entre personas jóvenes, generalmente las ubicamos fuera del contexto escolar, sin embargo, estos hechos amenazantes se viven frecuentemente en nuestras aulas, o con iguales en ambientes como: competencias intercolegiales, gimnasios, canchas de fútbol, y en los últimos años son una forma de protestar contra directores y docentes que imparten lecciones en escuelas y colegios.

La agresividad en los jóvenes costarricenses se está tornando en algo preocupante, al leer en los diarios titulares que señalan: "alumnos dañan oficina de colegio" o apedrean centros educativos (La Nación, 2007, Marzo 8). Estas situaciones lejos de promover la convivencia, alteran el desarrollo de la vida escolar.

Las conductas amenazantes e intimidantes, no pueden tomarse como parte del desarrollo normal, so pretexto de considerarlas una lucha entre iguales por obtener su identidad, o un espacio dentro del grupo. Si no se intervienen pronto y en forma adecuada, podrían ser el detonante 
de situaciones cada vez más violentas, que podrían dejar secuelas físicas y emocionales en nuestros estudiantes. Rodríguez y otros (2006) al respecto apuntan que:

"Las intimidaciones no se pueden considerar parte del común y normal desarrollo del adolescente; numerosos estudios las han relacionado con problemas emocionales y de conducta... y cada vez llegan más niños a consulta con cuadros diversos que tienen como trasfondo un maltrato en el colegio", p. 163.

Las alteraciones psicológicas no solo están presentes en las personas agredidas, sino también en quienes llevan a cabo esta agresión. Este fenómeno es conocido internacionalmente como "bullying", y los que participan de él son jóvenes escolares de ambos sexos (Olweus, 1994). Es importante señalar que no todos los comportamientos amenazantes pueden considerarse hostigamiento o maltrato; si estos problemas se analizan y solucionan dentro del grupo contribuyen al mejoramiento y desarrollo de las habilidades sociales entre los adolescentes.

Es claro que cada persona, sea maestro, director, personal de la escuela, padre o alumno, cumple un rol en toda situación de hostigamiento, por lo que debe llevarse a cabo una labor conjunta entre los participantes, buscando abordar con éxito esta problemática social.

\section{II. ¿Qué es el "bullying"?}

Una traducción literal de la palabra anglosajona "bullying" a la lengua española, señala que proviene de "bully" que significa matón o abusador y, como verbo: intimidar, amedrentar (ejercer influencia mediante la fuerza o coerción con el uso de un comportamiento o lenguaje de intimidación, mediante amenazas, insultos y un comportamiento agresivo) y abusar (tratar a otro en forma abusiva, o sea, usar mal, excesiva, injusta, impropia o indebidamente de alguien).

Se puede interpretar como el lastimar o aterrorizar a alguien quien es menor o menos poderoso, a menudo forzándolo a hacer algo que no quería. Como sinónimo tenemos "intimidate" (intimidar) que implica infundir miedo, temor o inducir un sentimiento de inferioridad en otra persona. En términos generales se da la intimidación y la superación de la resistencia generalmente mediante la insistencia, las exigencias, las amenazas y la fuerza. En la lengua española se tiene como sinónimo la palabra matonismo, que señala la conducta de quien quiere imponer su voluntad por la amenaza o el terror y que proviene de matón que literalmente significa hombre jactancioso y pendenciero, que procura intimidar a los demás. En esas circunstancias se da el acoso que implica perseguir, sin dar tregua ni reposo, a una persona. La persona es apremiada e importunada con molestias o requerimientos (American Heritage Dictionary, 2000, Cambridge Advanced Learner's Dictionary, Merriam-Webster Dictionary, Real Academia Española, 2001).

Internacionalmente se conoce como bullying a la conducta agresiva que se manifiesta entre jóvenes escolares. Esta conducta agresiva es deliberada y puede ocasionar daño físico y emocional. El fenómeno del maltrato por abuso de poder es también conocido como victimización en el entorno escolar o como acoso escolar.

Olweus (1998), uno de los primeros investigadores en estudiar el fenómeno del bullying, lo definió como:

la situación de acoso e intimidación, en donde un alumno es agredido o se convierte en víctima cuando está expuesto, de forma repetida durante un tiempo, a acciones negativas que lleva a cabo otro alumno o varios de ellos. p. 25 .

Con base en esta definición, se tienen tres criterios para identificar el "bullying".

Primero, un comportamiento agresivo con la intención de causar daño físico y psicológico, segundo, la reiteración de las conductas agresivas, se da incluso más allá del horario escolar, y tercero, la relación interpersonal se caracteriza por un 
desequilibrio de poder (real o superficial, de poder o fuerza) que hace a la víctima impotente para salir de esa situación por sí sola. Considerando este último punto se puede concluir que la victimización es un acto cobarde ya que quienes lo llevan a cabo saben de antemano que saldrán ilesos, puesto que la víctima se siente impotente para responder y difícilmente quienes observan esta conducta la denuncian (España. Defensor del Pueblo, 2007). El "bullying" puede tomar diferentes formas de presentación las que van desde el maltrato físico, verbal, psicológico, social, hasta los abusos sexuales.

De acuerdo con diferentes estudios (Olweus, 1993; Cerezo, 2006) se arremete contra un estudiante, cuando se dicen cosas inapropiadas de él, cuando se le amenaza, intimida o chantajea, se le quitan sus pertenencias, como el dinero, la comida; se le envían anónimos, se le insulta, se le golpea, se le empuja en los pasillos, y en algunos casos se le persigue fuera del recinto escolar.

La agresión física puede alcanzar dimensiones incontrolables en los centros educativos y ser mal conducida por las figuras de autoridad, llegando a convertirse los colegios en verdaderos centros de batalla, sobre todo si se cuenta con la permisividad e indiferencia por parte de las personas encargadas de disciplinar.

Viscardi (2003) llama al fenómeno de concebir algo como habitual "naturalización", y esto sucede en aquellos casos de niños durante el período de desarrollo, cuyas victimizaciones se generalizan al robo de pertenencias de sus compañeros, reciben palizas por parte de sus padres, o están expuestos a peleas tanto en el hogar como en el centro educativo. Este tipo de situaciones han llegado a percibirse como "normales" y que forman parte del desarrollo del niño. Si el acoso contra una víctima se da de manera frecuente y sostenida, podría relacionarse con una de las causas de deserción escolar, además de desencadenar problemas psicológicos, hasta conductas suicidas y homicidas.
"Un adolescente de 14 años de Hondarribia se suicidó, después de sufrir durante un tiempo el acoso y maltrato de varios de sus compañeros de clase. También recordamos todos el caso de los 2 niños británicos de 8 y 9 años que asesinaron a otro niño por el mero placer de "ver lo que sentía” y "qué cara ponía”. (Rodríguez y otros, 2006, p. 163)

Olweus (1998) anota una serie de indicios que presentan las personas a quienes se acosa, los que pueden observarse en los diferentes ambientes en que se desenvuelven:

a) En la escuela: son objeto de bromas de mal gusto, propensos a llamarles por un sobrenombre, sus compañeros se burlan en forma continua, les quitan sus meriendas, el dinero o les rompen sus pertenencias, entre otros. A estos indicios les llama primarios, por ser más directos y abiertos. Como indicios secundarios, anota aquellos utilizados para aislar a las víctimas, quienes generalmente se encuentran solas, sin amigos. También se puede observar que en los juegos participativos se les elige de último o no se les involucra. Pasan la mayor parte del tiempo en compañía de adultos, buscando protección, y presentan a la vez un bajo rendimiento escolar.

b) En el hogar: los indicios primarios son identificados por el padre, madre o figuras de crianza quienes observan que sus hijos regresan del colegio con sus pertenencias rotas, la ropa raída o sucia, con moretones y rasguños que no pueden explicar. Como indicios secundarios, los indicadores más frecuentes que se observan son: el que siempre están solos, no tienen compañeros, ni amigos que les visiten, son excluidos de las reuniones o fiestas con iguales, pierden interés por sus estudios, y muchas veces desertan del sistema educativo. Además de lo anterior, se suman otras características físicas que les distinguen como 
personas que se muestran más frágiles o menores que sus compañeros, su temperamento es más pasivo que activo, lloran con facilidad, les cuesta dar su opinión y cuando lo hacen, ésta no es escuchada por el grupo.

En cuanto a los agresores, generalmente participan gastando bromas pesadas, insultando, amenazando, ridiculizando, empujando o golpeando a su víctima. Por lo general, son físicamente más fuertes que sus compañeros, pueden ser de la misma edad o mayores que ellos. Tienen una gran necesidad de imponerse y dominar. Son populares en el grupo, pueden ser mayores que el resto de sus compañeros, y su rendimiento se encuentra dentro de los parámetros normales. (Olweus, 1998)

\section{Objetivo del trabajo}

El objetivo de esta investigación fue el de llevar a cabo un estudio piloto para detectar las conductas agresivas que presentan estudiantes de seis centros educativos costarricenses con edades entre los 13 y 16 años, y elaborar un perfil de las conductas amenazantes que se presentan con mayor frecuencia.

\section{Metodología}

El trabajo se efectuó con una muestra de 371 alumnos de ambos sexos, con edades entre los 13 y 16 años, ubicados en los niveles de $6^{\circ}, 7^{\circ}$ y $8^{\circ}$ del sistema educativo costarricense, en las zonas de Coronado, Cartago, Alajuela y Alajuelita.

Se le explicó a cada uno de los grupos de qué trataba el estudio, se les motivó para contestar un cuestionario, y se les entregó un formulario que debían resolver en forma individual y anónima, lo que permitió que sus respuestas se dieran en forma más abierta.

\section{a) Instrumento}

El cuestionario que se aplicó se divide en dos partes. La primera parte trata de detectar el número de estudiantes que se ha visto involucrado en conductas agresivas dentro del aula, como iniciadores o agresores. Pretende detectar ¿cómo se lleva a cabo la agresión?, ¿qué características presentan las personas que son agredidas?, ¿con qué frecuencia se presentan las conductas agresivas en los centros educativos?, ¿cómo reaccionan las figuras de autoridad ante estos hechos?, y ¿qué sentimientos se generan en las personas que cometen estos actos? La segunda parte, pretende detectar si la muestra encuestada ha sido víctima de intimidaciones y cuyos datos no serán analizados en este primer informe.

\section{Análisis de los datos}

Los datos se analizan en forma porcentual. Para ello se elaboró una tabla con las variables que se quieren medir, contabilizándose cada respuesta de los estudiantes.

Las primeras cuatro preguntas pretenden medir las diferentes conductas de las personas que participan de acciones de acoso en los centros educativos, las formas en que lo hacen, las características de las personas a quienes se victimiza, y las razones que privan para agredir a otras personas.

De acuerdo con la literatura (Olweus, 1998), las variables estudiadas, están estrechamente relacionadas con la dinámica de bullyings.

Las preguntas 5 a 7 son más situacionales, y permitieron obtener información sobre el lugar en que se dio la agresión, si las personas estaban solas o acompañadas, y con qué frecuencia participaron los estudiantes en ella. La pregunta 8 mide los sentimientos del agresor, una vez que intimidan a su víctima. 


\section{Resultados y discusión}

Los resultados de este estudio, se presentan en tablas en función del género. De acuerdo con los datos obtenidos se estima que un $19,1 \%$ de los estudiantes de los seis centros educativos costarricenses que participaron, cuyas edades están entre los 13 y 16 años, se han visto involucrados en actos de acoso. Estos datos pueden compararse con la media europea cuyos porcentajes se encuentran entre el $15 \%$ y el $20 \%$ de los casos, países dentro de los que están Noruega y España. Ver Tabla 1.

Tabla 1

Porcentajes de participación en conductas amenazantes de estudiantes costarricenses

\begin{tabular}{ccc}
\hline Varones & Mujeres & Total \\
\hline 9,7 & 7,4 & 17,1 \\
\hline
\end{tabular}

Las formas más frecuentes de acosar en este grupo se dan a través de los golpes, el uso de palabras fuertes, grotescas y salidas de tono, hasta llegar a la intimidación mediante cuchillos, punzones, y armas de fuego. Entre los varones el acoso físico se presentó con mayor frecuencia mientras que en las mujeres, principalmente como una forma de chantaje y de hostigamiento más sutil, mediante la intimidación de su víctima, bajo la amenaza de "divulgar un secreto" o "decir algo que le avergonzaría a su compañero(a)", manipulando de esta forma la relación de amistad existente. El acoso verbal y el uso de palabras fuertes, es la forma más común en ambos grupos, datos que fueron corroborados por las respuestas dadas por los estudiantes y que se presentan en Tabla 2.

Tabla 2

Porcentajes de las formas de acoso

\begin{tabular}{lcc}
\hline \multicolumn{1}{c}{ Respuesta } & Varones & Mujeres \\
\hline Golpear & 2,7 & 1,1 \\
Amenazas de palabra & 4,9 & 6,7 \\
Uso de palabras fuertes & 1,1 & 1,1 \\
Amenaza con cuchillo & 2,8 & 0,1 \\
Total & 11,5 & 9,0 \\
\hline
\end{tabular}

Como principales características anotadas por los estudiantes para referirse a las personas a quienes acosan (víctimas) se dieron los calificativos de "tontas", "débiles", "que no reaccionan" y "fáciles de manipular" entre otras. El agresor percibe a sus víctimas como personas frágiles y pasivas, lo que facilita sus acciones ya que se convierten en un blanco fácil, porque no le denunciarán ante sus profesores por temor ante su victimario. Esta caracterización coincide con los resultados de las investigaciones de Olweus (1996) quien ha encontrado que el tipo más frecuente de víctimas suelen ser prudentes, sensibles, calladas, apartadas, tímidas, inseguras, tristes, de baja auto estima, en el caso de las mujeres. En el caso de los varones suelen ser más débiles que sus compañeros. La característica de debilidad es percibida por las mujeres encuestadas en un $51 \%$, mientras que en los varones en un $10 \%$, y un $13 \%$ justifican sus reacciones al decir que fueron provocados con anterioridad. En la Tabla 3 se anotan las principales características encontradas en ambos grupos encuestados.

Tabla 3

Características de las personas acosadas

\begin{tabular}{ll}
\hline Varones & Mujeres \\
\hline No fue en serio & Porque es más fácil \\
Porque es un tonto & Porque era tímida \\
No sabe & Porque es manipulable \\
Porque lo vi más débil & Es más pequeña que yo \\
que yo & No sé \\
Porque me cae mal & Me cae mal \\
Porque era menor & No lo escogí \\
Porque no reacciona & Por ser ella \\
Se me presentó & Traté de asustarla \\
& Ofendió a mi mamá \\
\hline
\end{tabular}

Las diversas formas de relacionarse de los adolescentes, están dejando de ser algo natural de compartir. En nuestro país las conductas amenazantes, en los jóvenes, están tomando una escalada ascendente a la que debe prestarse más atención. 
Para los costarricenses ya no es extraño leer informaciones como "Alumnos dañan oficina de colegio en Desamparados" (La Nación, 2007, Marzo 8) o que "alumnos de un Liceo apedrean a otro colegio". (La Nación, 2007, Marzo 13)

Este tipo de agresiones, lejos de ser una situación normal entre iguales, han pasado a convertirse en algo preocupante, que afecta a todos los grupos, y pueden desencadenar situaciones emocionales conflictivas que desestabilicen a los alumnos, y a futuro a la sociedad misma. Estas conductas intimidantes fueron justificadas por respuestas como: no fue en serio, porque tenía algo que yo necesitaba, porque me gusta hacerlo, porque era lo más cerca que tenía, me cae mal, para que no me molestara, me debía plata, me insultó, me buscó, en el caso de los varones, y en el caso de las mujeres las justificaciones fueron: para que me dejara, le gustaba mi pareja, me molestaba, me cae mal, me amenazó, me dio cólera, insultó a mi mamá, entre otras.

Las intimidaciones no pueden considerarse parte del común y normal desarrollo del adolecente; numerosos estudios las han relacionado con problemas emocionales y de conducta. (Rodríguez y otros, p. 163)

En el caso de las mujeres, en la muestra costarricense, las razones dadas son diferentes; al analizar las respuestas pareciera que medió la provocación ("para que no me amenazara", "que no me acose", "tenía algo mío", "me critica", "me molestan" entre otras). En los varones los motivos son variados, los que van desde el maltrato físico, intencional, hasta por el placer de hacerlo obteniéndose respuestas como: "por sentir la adrenalina", "por nada", "porque quería hacerlo". Situaciones a las que debe prestarse especial atención ya que podrían llevar a un trastorno antisocial de la personalidad, que lleva a: "un patrón general de desprecio y violación de los derechos de los demás, que comienza en la infancia o el principio de la adolescencia y continúa en la edad adulta" (DSM-IV, 1995, p. 662). Estas personas no logran adaptarse a las normas y reglas sociales, lo que eventualmente les conduciría a cometer actos, que ameriten una detención.

En la tabla 4 se anotan las diversas razones que dieron ambos grupos acerca de qué fue lo que les impulsó a llevar a cabo esa agresión.

Tabla 4

Razones que impulsaron a la agresión

\begin{tabular}{ll}
\hline Varones & Mujeres \\
\hline Por vacilar & Por mi inteligencia \\
Ninguna & Porque me joden demasiado \\
No me hace caso & Porque solo quería una cosa \\
Me molesta & No me hace caso \\
Me golpeó & No se podía evitar \\
Necesitaba plata & Quería saber mi secreto \\
Me cae mal & Para amenazarla \\
Me debía plata & Que no me delatara \\
Cólera & Para que no me amenazara \\
Juega de vivo & Me coge las cosas \\
Me quitó un lapicero & Que no me acose \\
Me molestaba & Tenía algo mío \\
No me respeta & Me cae mal \\
Para pasar el año sin & Me critica \\
dificultades & \\
Nadie debía saberlo & Me tenía asqueada \\
Por pasivo & Me provocó \\
Me insultó & Juega de viva \\
Resentimiento & Me “enjacha" \\
Por miedo & Por defender a mi mamá \\
Para que me diera lo & Me molestan \\
que quería & \\
Por la adrenalina & Por cólera \\
\hline
\end{tabular}

Los datos obtenidos muestran que en un $61,1 \%$ de las veces, los varones se encontraban acompañados, y en el caso de las mujeres en un $46,3 \%$ se encontraban solas. El fenómeno del agrupamiento según Olweus $(1978,1998)$ es una respuesta a los mecanismos socio culturales establecidos lo que lleva al contagio social, a una debilitación del control, a la división de la responsabilidad, además de cambios graduados cognitivos de la percepción del acosador y la víctima, lo que a la vez explica, el por qué hay estudiantes que no son agresivos, 
pero participan en forma pasiva también del acoso. La intimidación por parte de las mujeres, en un porcentaje muy alto se da cuando se encuentran solas con su víctima, mientras que en los varones, cuando se encuentran acompañados, ver Tabla 5.

Tabla 5

Situación en que se dio el acoso

\begin{tabular}{|l|c|c|c|}
\hline & $\begin{array}{c}\text { Estaba } \\
\text { solo(a) }\end{array}$ & $\begin{array}{c}\text { Estaba } \\
\text { acompañado(a) }\end{array}$ & total \\
\hline Varones & $38,9 \%$ & $61,1 \%$ & 100 \\
\hline Mujeres & $53,85 \%$ & 46,15 & 100 \\
\hline
\end{tabular}

Llama la atención la poca vigilancia que hay y como consecuencia las pocas medidas correctivas aplicadas, en los centros educativos y el hogar, como puede apreciarse. En un 50,6\% de las veces en que ambos grupos agredieron a sus compañeros de una u otra forma, no fueron castigados, ni recibieron consecuencias que corrigieran esa conducta. De este grupo de estudiantes solamente un 5,9\% de los varones fue amonestado con una boleta, mientras que solamente a un $16,2 \%$ de las mujeres se les regañó, situaciones que no cambiaron las conductas amenazantes. Los datos obtenidos pueden verse en las Tablas 6 y 7 .

Tabla 6

Consecuencias de conductas amenazantes en varones

\begin{tabular}{lc}
\hline Conducta & Porcentajes \\
\hline Me lo devolvió & 2,9 \\
Nadie se dio cuenta & 29,4 \\
Regaño & 11,8 \\
Nada lo hice en broma & 5,9 \\
Amenacé de nuevo & 2,9 \\
Conseguí lo que quería & 2,9 \\
Lo suspendieron & 2,9 \\
Amigo se enojó & 2,9 \\
Lo amonestaron & 5,9 \\
Pidió disculpas & 2,9 \\
Nada & 29,4 \\
Total de conductas sin corregir & 61,8 \\
\hline
\end{tabular}

Tabla 7

Consecuencias de conductas amenazantes en mujeres

\begin{tabular}{lc}
\hline Conducta & Porcentajes \\
\hline Nadie se dio cuenta & 21,6 \\
Castigo & 18,9 \\
Consiguió lo que quería & 2,7 \\
Nada & 37,8 \\
Regaño & 16,2 \\
Boletas & 2,7 \\
Total de conductas sin corregir & 59,5 \\
\hline
\end{tabular}

La frecuencia con que los varones agreden a sus víctimas es alta. Un 16\% lo hace diariamente, un $27,9 \%$ una vez por semana. Si sumamos ambos criterios podríamos decir que un $43,9 \%$ lo hace semanalmente. Este indicador nos lleva a pensar que las relaciones cordiales fundamentales para la convivencia entre iguales, se sustituye por la fuerza y la intimidación, en ambientes que sirven de modelo para una inserción social adecuada. Si este comportamiento se da en forma repetida e intencional contra una víctima puede causar daño, y si el mismo se extiende fuera del ámbito escolar, los resultados podrían ser devastadores, porque en muchas ocasiones, se combina la violencia física con la violencia psicológica.

En el ámbito escolar, al ser el acosador o la víctima, parte de un grupo, la situación no solo se circunscribe a una persona, sino que al resto de sus compañeros, quienes directa o indirectamente ejercen en alguna medida influencia en esta situación. Si el resto de los compañeros se adhieren en forma pasiva al acosador, para la víctima se convierte en un proceso humillante, que socava su autoestima, aumenta la sensación de inseguridad y temor en el Centro Educativo, provocando en muchos casos la deserción como una forma de protegerse.

El fracaso escolar, no solo debe medirse a través del alcance de aquellos objetivos académicos no logrados por los educandos, 
sino por estas otras situaciones que muchas veces escapan al conocimiento de los docentes.

En la Tabla 8 se anota la frecuencia con que se presentaron las conductas amenazantes en la muestra seleccionada.

Tabla 8

Frecuencia con que se presentan las conductas amenazantes

\begin{tabular}{lcc}
\hline Tiempo & Varones & Mujeres \\
\hline Diariamente & 16,6 & \\
Una vez por semana & 27,9 & 12,1 \\
Una vez por mes & 16,6 & 21,2 \\
Una vez al año & 34,9 & 66,7 \\
\hline
\end{tabular}

Son diversos los sentimientos que se generaron en los estudiantes que agredieron a sus compañeros y van desde la culpabilidad, el sentirse felices, avergonzados, con ganas de venganza y matar, hasta la total indiferencia.

Ambos grupos dieron más de una respuesta, por lo que los porcentajes se dan en función del número de respuestas y no del número de sujetos.

Las sensaciones de satisfacción, poder, venganza y ganas de matar, son particularmente descriptores del matonismo, y a la vez conductas antisociales que aparecen claramente en la muestra estudiada. La intencionalidad está presente, a través del deseo de "matar" y de "venganza" manifestaciones que se refuerzan con las sensaciones de "felicidad", "satisfacción" y "poder" que experimentan los jóvenes. La agresión es una forma de conducta cuya intención es herir física o psicológicamente a alguien (Berkowitz, 1993). En la Tabla 9 se anotan los sentimientos generados en el grupo estudiado.

\section{Conclusiones}

El Bullying como una conducta agresiva, amenazante, que se presenta entre la población escolar, es un fenómeno mundial, y no escapa a las aulas de los estudiantes costarricenses. Se presenta en forma sostenida a través de la vida escolar de muchos de nuestros educandos lo que les afecta de una u otra manera, entorpeciendo su desarrollo emocional y académico en el sistema educativo.

La presencia de conductas amenazantes en los estudiantes de los Centros Educativos costarricenses es más frecuente de lo esperado. Los resultados obtenidos indican que un $17,1 \%$ de los escolares ha participado en algún tipo de agresión, lo que es comparable con los datos europeos que oscilan entre un 15 y un $20 \%$.

Diversos estudios (Ortega, 1994; Olweus, 1998; Cerezo, 2006) han encontrado

Tabla 9

Sentimientos generados por la agresión

\begin{tabular}{lcrr}
\hline Sentimientos & Varones & Mujeres & Total \\
\hline Culpable & 29,5 & 25,0 & 54,5 \\
Feliz & 3,3 & 2,3 & 5,5 \\
Poderoso & 11,5 & 9,1 & 20,6 \\
Avergonzado & 4,9 & 11,4 & 16,3 \\
Satisfecho & 14,8 & 15,9 & 30,7 \\
Preocupado & 21,3 & 25,0 & 46,3 \\
Con ganas de matar & 1,6 & & 1,6 \\
Venganza & 3,3 & & 3,3 \\
Enojado & 4,9 & 2,3 & 7,2 \\
Nada & 4,9 & 11,4 & 16,3 \\
\hline
\end{tabular}


que existe una relación de 3:1 (tres hombres por cada mujer) de estudiantes que participan de conductas intimidantes en los centros educativos, en diversas partes del mundo, sin embargo, esta relación en la muestra costarricense no es tan marcada. Los datos encontrados nos muestran que tanto los varones como las mujeres participan arremetiendo contra sus iguales casi en una misma proporción (9,4\% mujeres, 9,7\% varones). Estos porcentajes se salen de los parámetros establecidos por estudios previos en poblaciones diferentes, lo que llama a la reflexión y a repetir este estudio con una muestra más grande, que dé indicadores del ¿por qué se establece esta relación?

Una cantidad muy alta de estudiantes que arremete contra sus iguales, no recibe ningún castigo, situación de alerta que llama la atención hacia aquellas personas encargadas de disciplinar cuyo rol no se está cumpliendo. Esto se desprende de los datos suministrados por el grupo encuestado en el que un $29,4 \%$ de los varones manifestó que nadie se dio cuenta y ese mismo porcentaje $(29,4 \%)$ indicó que no recibió ningún castigo. En el caso de las mujeres, un $21,6 \%$ anotó que nadie se dio cuenta de lo sucedido por lo que ellas tampoco recibieron ningún castigo. $\mathrm{Si}$ sumamos ambos porcentajes tenemos que en un $51 \%$ de las veces las personas que arremetieron contra otros, no recibieron, ninguna consecuencia, por sus actos, por lo que la conducta de agredir a otros se ve reforzada, al no aplicarse medidas correctivas que impidan esta acción.

Las formas más frecuentes de agresión en la muestra costarricense se manifiestan del siguiente modo:

a) El maltrato físico que va desde los golpes, empujones, hasta amenazas con cuchillos, punzones, y armas de fuego.

b) El maltrato verbal que se presenta mediante el uso palabras soeces, insultos, que humillan y descalifican a las víctimas. c) Las amenazas y el chantaje que se llevan a cabo diciendo a la persona que revelará un secreto suyo, o que propagará algún rumor, manteniendo de esta forma el dominio de la situación, como una muestra de poder y sometimiento de la víctima.

d) La amenaza verbal es la más utilizada, y esta se ve favorecida por las características propias de las víctimas quienes son percibidas como personas "más tímidas", "más pequeñas", "más tontas", o "que no reaccionan", rasgos que las convierten en un

blanco disponible en el qué descargar la hostilidad cuando la persona que la provoca no puede ser contestada: el niño es un chivo seguro de atacar y está garantizado que no contraatacará. En el marco escolar un blanco fácil es el débil de la clase, aquel que tiene algún tipo de "hándicap" o discapacidad. (Olweus, 1998, p.64)

Los rasgos encontrados en el grupo de estudiantes costarricenses, son los mismos encontrados en otras poblaciones ya estudiadas, de allí que las víctimas se describan como

aquellos alumnos más ansiosos e inseguros que el resto. Además suelen ser cautos sensibles y tranquilos. Cuando se sienten atacados normalmente reaccionan llorando (al menos en los cursos inferiores) y alejándose. Así mismo padecen una baja autoestima, y tienen una opinión negativa de sí mismos y de su situación. (Olweus, 1998, p. 50)

El bullying en las mujeres costarricenses se presenta bajo una forma más sutil, pero las consecuencias para las víctimas son igualmente destructivas.

Se inicia en la escuela bajo el consentimiento de los adultos o figuras de autoridad, al interpretarse estas relaciones como inocentes, y permisivas en el salón de clases, cuando el niño es pequeño y despoja a otro de sus lápices o raya su cuaderno en forma sistemática, atemorizándole; y continúa en la adolescencia, con otros indicadores más abiertos como el empujar, golpear e insultar a sus 
compañeros, hasta llegar a las amenazas con cuchillos y armas de fuego.

Al no existir una legislación que permita castigar estos actos, en personas menores de edad, que se tornan cada vez más agresivas y desafiantes para los grupos sociales, y al no abordase el problema con una intervención inmediata, la escalada irá en aumento hasta alcanzar niveles que serán difíciles de manejar por los docentes, padres y otras figuras de autoridad.

Con frecuencia no se aborda esta problemática a través de programas comprensivos, para prevenir se desencadene este fenómeno, sino que tratamos de intervenir cuando la problemática está en el aula. Una posible intervención podría iniciar:

a. Con una toma de conciencia por parte de quienes educan, al aceptar que el problema es real, que afecta a un porcentaje considerable de la población escolar, que en ocasiones es causa de la deserción escolar en muchas instituciones educativas, y que esta problemática afecta a los jóvenes, provocando sentimientos de miedo, ansiedad, inseguridad, en una etapa de formación, en donde deberían estar disfrutando del aprendizaje, la comunicación y convivencia con iguales.

b. Elaborando un listado de conductas que se consideren agresivas, a través de la aplicación de instrumentos que permitan detectar diferentes perfiles de los agresores, así como los focos de discordia, las características socio afectivas del grupo, y otros aspectos como los anotados en esta investigación.

c. Desarrollando un programa conductual en donde se utilicen diversas estrategias correctivas apoyadas en el reforzamiento, economía de fichas y contratos, para ser aplicadas dentro y fuera del aula, específicamente con aquellos estudiantes que han sido identificados en el salón de clase como agresores o víctimas, permitiendo la participación activa a su vez, de todos los alumnos.

d. Promoviendo el diálogo y la confianza con el docente, que conduzca a que las víctimas rompan su silencio, a través de dinámicas de grupo, en donde se compartan experiencia que faciliten este proceso.

\section{Referencias bibliográficas}

Alumnos dañan oficina de colegio en Desamparados. (2007, Marzo 8). La Nación, p. 16.

Alumnos del Liceo de Costa Rica apedrean a otro colegio. (2007, Marzo 31). La Nación, p. 10.

American Heritage Dictionaries. (2006). (Ed.). American Heritage Dictionary of the English Language ( $4^{\mathrm{a}}$ ed., Nueva York: Houghton Mifflin.

Berkowitz, L. (1993). Aggression: Its causes, consequences, and control.Philadelphia: Temple University Press.

Boute, C. A. (2001). Relations of autonomy and relatedness to school functioning and psychological adjustment during adolescence. Disertation Abstracts International Section A: Humanities and Social Sciences, 62(1).

Cambridge Advanced Learner's Dictionary. http://dictionary.cambridge.org

Castejón, J. L. y Pérez A. M. (1998). Un modelo causal explicativo sobre la influencia de las variables psicosociales en el rendimiento académico. Revista el Bordón, 50(2), 171-175.

Cerezo, F. (2006). Violencia en las aulas. Madrid: Pirámide. 
Cerezo, F. (2006). Conductas agresivas en la edad escolar. Madrid: Pirámide.

España. Defensor del Pueblo. (2007). Violencia escolar: el maltrato entre iguales en la Educación Secundaria Obligatoria 1999-2006. Madrid: Defensor del Pueblo.

Manual diagnóstico y estadístico de los trastornos mentales (DSM-IV). (1995). España: Masson, S.A.

Merriam-Webster on Line Dictionary. http://www.merriam-webster.com/ dictionary

Ortega, R. (1994). Violencia interpersonal en los centros educativos de enseñanza secundaria. Un estudio sobre el maltrato y la intimidación entre compañeros. Revista de Educación. No. 304, 55-67.

Olweus, D. (1983). "Low school achievement and aggressive behaviour in adolescent boys". En Magnusson, D. y Allen, V. (Eds.). Human development. An interactional perspective. Nueva York: Academic Press, pp. 353-365.

Olweus, D. (1994). "Bullying at school: Basic facts and effects of a school based intervention program". J. Child Psychol. Psychiatry 35: 1171-90.

Olweus, D. (1996). The Revised Olweus Bully/Victim Questionnaire. Bergen: Maneo.
Olweus, D. (1978). Agression in the schools: Bullies and whipping boys. Washington D.C.: Hemisphere Publ. Corp.

Olweus, D. (1980). Familiar and temperamental determinants of aggressive behavior in adolescent boys: A causal analysis. Developmental Psychology, 16, 644-660.

Olweus, D. (1998). Bullying at school: What we know and what we can do. [Edición en español: Conductas de acoso y amenaza entre escolares]. Madrid: Ediciones Morata.

Real Academia Española. (2001). Diccionario de la lengua española. $\left(22^{\mathrm{a}} \mathrm{ed}\right.$. Vol. 2). Madrid: Espasa-Calpe, S.A.

Rodríguez, R., Seoane, A. y Pereira, J. L. (2006). "Niños contra Niños: El bullying como trastorno emergente". Anal Pediátrico (Barcelona). 64(2): 162-166.

Serrano, I. (2006). Agresividad Infantil. Madrid: Pirámide.

Viscardi, N. (2003). Enfrentando la violencia en las escuelas: un informe de Uruguay. En UNESCO, autorii, varii, Barsil. Violencia na Escola: América Latina e Caribe Brasilia, Brasil: Ediciones UNESCO, 153-205. 
